\title{
Prática do abortamento entre adolescentes: um estudo em dez escolas de Maceió (AL, Brasil)
}

\author{
Practice of abortion among teenagers: \\ a study in ten schools of Maceió (AL, Brazil)
}

Divanise Suruagy Correia ${ }^{1}$

Jairo Calado Cavalcante ${ }^{1}$

Eryvaldo Sócrates Tabosa do Egito ${ }^{2}$

Eulália Maria Chaves Maia ${ }^{2}$

${ }^{1}$ Faculdade de Medicina, Universidade Federal de Alagoas. Campus A. C. Simões, Tabuleiro dos Martins. 57072-900 Maceió AL.

divanisesuruagy@yahoo.com.br ${ }^{2}$ Programa de Pós-

Graduação em Ciências da Saúde, Universidade Federal do Rio Grande do Norte.

\begin{abstract}
This is a cross-cut study that was carried out with the objective of investigating the causes through which adolescents have provoked abortion, relating it to age and the type of school attended. The sample was calculated by taking into account the number of hospital admissions for post-abortion curettage. A semi-structured and anonymous questionnaire was used as a research instrument which was applied in ten schools randomly chosen among all schools, of Maceió (Alagoas, Brazil), to reach 12-19 years old female teenagers. The data were assessed by the Epi Info Program making use of odds ratio and a relative risk to verify any association among variables and a confidence interval at $95 \%$. At a sample of 2,592 adolescents, $559(21.6 \%)$ had an active sexual life, $182(7.0 \%)$ informed to have been pregnant and $149(26.7 \%)$ to have aborted. The fear of the parents' reaction, age, lack of support of the partner and pregnancy rejection were the explanation to stimulate abortion. Fear was the most mentioned cause in both types of school. Abortion was more mentioned in public schools, being significant and protective the risk of aborting before the age of 15 . The fear of the parents' reaction as a frequent cause suggests the necessity for additional studies on sexuality and communication between parents and children.
\end{abstract}

Key words Sexuality, Adolescent, Abortion
Resumo Trata-se de um estudo de corte transversal, realizado com o objetivo de investigar as razões que levaram adolescentes a provocarem o aborto, relacionando com idade e tipo de escola que frequentavam. A amostra foi calculada considerando o número de internações para curetagem pós-abortamento. Usou-se como instrumento um questionário semiestruturado, anônimo, aplicado em dez escolas, sorteadas dentre todas da cidade de Maceió (Alagoas), pesquisando-se adolescentes dos 12 aos 19 anos, do sexo feminino. Os dados foram analisados pelo Programa Epi Info, usando-se odds ratio e risco relativo para verificar associação entre variáveis e intervalo de confiança a 95\%. Em uma amostra de 2.592 jovens, 559 (21,6\%) tinham vida sexual ativa, 182 (7,0\%) referiram ter engravidado e 149 (26,7\%) abortado. Medo da reação dos pais, idade, falta de apoio do companheiro e rejeição da gravidez foram razões para provocar $o$ aborto, sendo medo a mais citada, em ambos os tipos de escola. O aborto foi mais citado nas escolas públicas, sendo significativo e protetor o risco para abortar antes dos 15 anos, e significativa a relação entre abortar e estudar em escolas públicas. O medo da reação dos pais como razão mais frequente sugere a necessidade de novos estudos sobre sexualidade e comunicação entre pais e filhos.

Palavras-chave Sexualidade, Adolescente, Aborto 


\section{Introdução}

A adolescência é a fase da vida entre a infância e a fase adulta em que ocorrem transformações biológicas, socais e mentais. A Organização Mundial da Saúde (OMS) considera esta fase como a segunda década da vida, compreendida entre 10 e 19 anos. A juventude, por sua vez, é a fase que se estende dos 15 aos 24 anos ${ }^{1}$.

É reconhecida a importância dos aspectos sociais na saúde de uma população. A saúde do adolescente destaca questões como a formação familiar e o nível educacional, que interferem em diversos aspectos de sua saúde. Ações realizadas nesse âmbito se refletirão preventivamente no controle dos riscos à saúde, comuns nessa fase de desenvolvimento, tais como doenças sexualmente transmissíveis, gravidez não planejada, uso de drogas lícitas e ilícitas e acidentes de trânsito ${ }^{1,2}$.

O exercício da sexualidade sem a devida maturidade e responsabilidade mostra seus resultados na incidência de nosologia relacionada a esse comportamento, bem como a gravidez não planejada ${ }^{3-6}$. Segundo Cabral ${ }^{6}$, no Brasil, alguns fatores estão relacionados com o aparecimento da gravidez nesse período: a monoparentalidade feminina, a precariedade socioeconômica, a interrupção do processo de escolarização e as dificuldades de inserção profissional.

Estudos realizados no Brasil confirmam a desinformação ou o baixo nível de conhecimento sobre saúde reprodutiva pelas adolescentes, $o$ que alerta para uma educação sexual inadequada. Sabe-se que quase não há, no país, serviços de saúde disponíveis para atender especificamente às necessidades próprias dos adolescentes, o que se configura em um potencial obstáculo para o acesso às informações e às ações que protejam a saúde desses jovens ${ }^{1,3,4,7}$.

Dessa forma, a sexualidade da juventude tem despertado a atenção para a necessidade de políticas públicas no Brasil. Tem-se destacado a necessidade de atenção à faixa etária dos 10 aos 15 anos, na qual o número de gestações não segue a tendência de queda do resto da população ${ }^{3}$.

Por sua vez, o fenômeno da gravidez não planejada nesse período traz a reflexão sobre o aborto provocado, que apesar de proibido por lei, no Brasil, é frequentemente realizado na adolescência. Pesquisas mundiais mostram que o número de abortamento espontâneo não é diferente de uma população para outra, no entanto os percentuais referentes aos abortos induzidos são divergentes entre os países e diversos grupos sociais e étnicos ${ }^{4-8}$.
Atualmente, os países desenvolvidos apresentam uma diminuição nos números de gravidez e abortamento provocado na adolescência; todavia, nos países em desenvolvimento, os estudos mostram uma alta prevalência de abortos e baixo uso de métodos contraceptivos nessa fase. Estima-se que ocorrem a cada ano 46 milhões de abortos provocados em todo o mundo, e cerca de 20 milhões são clandestinos ${ }^{7,8}$.

Nos locais onde o aborto é permitido por lei, os registros são mais confiáveis do que nos lugares onde o ato é ilegal, e mesmo nos países onde os métodos contraceptivos estão facilmente disponíveis, o número de gravidezes não planejadas é alto, e parte delas termina em abortamento provocado $^{7-11}$.

Esse é um problema de Saúde Pública na America Latina, e as estatísticas sobre mortalidade relacionadas com hospitalizações o assinalam como uma das principais causas de morte de mulheres no Brasil. Tal fato demonstra as desigualdades sociais brasileiras, uma vez que as jovens que frequentam os hospitais públicos, em busca de procedimentos médicos após tentativa de abortamento, são aquelas mais expostas à exclusão social ${ }^{1,3,11-13}$.

Atualmente, o óbito de mulheres por causas ligadas à gravidez, ao parto e ao puerpério é em grande parte prevenível. A mortalidade materna é um indicador de saúde que demonstra as condições de vida da população e a qualidade da atenção à saúde da mulher. Por sua vez, o acesso aos serviços de saúde e a concentração de renda são alguns dos determinantes para esse problema ${ }^{14}$.

No ano de 2004, Maceió mostrou que 86\% das adolescentes grávidas eram solteiras ${ }^{12}$, o que as coloca diante dos conflitos de uma gravidez solitária, afora aqueles próprios da idade ${ }^{1}$. Sabese que são várias as razões que levam uma mulher a provocar um aborto. Geralmente elas estão associadas com idade, fator socioeconômico e número de filhos. As adolescentes o praticam porque são ainda estudantes, dependentes economicamente dos pais, ou foram abandonadas pelos companheiros ${ }^{5-10}$.

A magnitude do problema abortamento provocado nessa população e os poucos trabalhos realizados justificam o presente estudo, que teve como objetivo investigar as razões que levaram adolescentes estudantes de Maceió a provocarem o aborto, relacionando-o com a idade e o tipo de escola que frequentavam, sendo parte de uma pesquisa maior sobre causas de abortamento. 
Trata-se de um estudo de corte transversal, exploratório, realizado em dez escolas da cidade de Maceió, no ano de 2005. O cálculo da amostra para estudos sobre aborto provocado em países onde o ato é ilegal apresenta dificuldades ante a subnotificação. Neste estudo, consideraram-se as recomendações de Rossier ${ }^{15}$ e do Allan Guttmacher Institute ${ }^{5}$, e calculou-se a amostra levandose em conta o número de internações para curetagem pós-abortamento, obtidos do Sistema de Informação de Internação Hospitalar-Datasus ${ }^{13}$. Este estudo faz parte de outro maior sobre abortamento provocado em Maceió.

Segundo esse Sistema ${ }^{13}$, em Maceió, o ano de 2004 apresentou 741 partos para a idade dos 10 aos 14 anos e 13.857 dos 15 aos 19 anos de idade. Para uma população feminina de 344.221 adolescentes, de 10 a 19 anos de idade, mostrou 1.327 internações, de um total de 5.622. Estes números resultam numa prevalência de 9,81\% de curetagens, prevalência esta que foi usada (10\%) para calcular a amostra representativa para abortamento, neste estudo.

Usando-se esses dados, o Programa Epi Info, com $\mathrm{IC}_{95 \%}$ e a prevalência de $10 \%$, foi realizado um cálculo amostral que apontou um n de 864 gestações para a faixa etária estipulada. Usandose então a prevalência encontrada, que coincide com a prevalência máxima sugerida pelos estudos para cálculo de pesquisas sobre abortos ${ }^{15,16}$, observou-se a necessidade de se pesquisar no mínimo 86 adolescentes que tivessem praticado o ato, a fim de obter resultados representativos.

Para confirmar esse cálculo amostral, e continuando a usar a literatura estudada ${ }^{6,15,16}$, considerou-se o número total de curetagens pósaborto (5.622), corrigiu-se o valor em $12 \%$, considerando as subestatísticas, deduziram-se 25\% do dado encontrado, levando em conta os abortos espontâneos, multiplicou-se pelo fator de correção cinco sugerido para o Brasil ${ }^{17}$ e encontrou-se uma amostra de 2.530 adolescentes.

Para o sorteio das escolas, foram selecionadas da rede de ensino público e particular da cidade de Maceió todas aquelas que possuíam os níveis de ensino fundamental e médio, observando-se o número total de alunos matriculados em cada uma delas, no ano de 2004, visando ao alcance da amostra na faixa etária selecionada. Estimou-se que 50\% delas seriam do sexo feminino, e calculou-se por faixa etária o número a ser encontrado em cada uma delas. Constatou-se então que o número de dez escolas seria suficiente para a obtenção da amos- tra estabelecida. A partir disto, foi realizado o sorteio das instituições de ensino, considerando-se as $50 \%$ para rede pública e 50\% para a particular. Foi necessário repetir o sorteio três vezes, entre as escolas particulares, porque algumas direções não autorizaram a coleta dos dados em seus estabelecimentos. Não houve problema na rede pública.

Os dados foram obtidos através de um questionário semiestruturado subdividido em três blocos básicos de dados: Sociodemográfico, Vida Sexual e Gravidez/Aborto. Apresenta-se neste estudo o resultado de uma das perguntas, que foi: "O que levou você a praticar o aborto?" O instrumento foi aplicado em sala de aula, em momento específico para tal, após o preenchimento do Termo de Consentimento Livre e Esclarecido pela jovem, e a obtenção do Termo dos respectivos pais ou responsáveis. Foi realizado um estudo piloto para adequação do instrumento, e a coleta de informações aconteceu no ano de 2005.

A composição da amostra a partir dos 12 anos de idade, e não dos 10 anos, início da adolescência, preconizado pela Organização Mundial da Saúde, prendeu-se ao fato do interesse das jovens em responder ao questionário, bem como da autorização dos seus responsáveis, quando da operacionalização da coleta de dados.

$\mathrm{Na}$ amostra de 2.592 jovens, foram encontradas $559(21,6 \%)$ com vida sexual ativa, que são aqui estudadas. Os dados apresentaram uma distribuição normal, com idade média e mediana de 15 anos, 1,5 de desvio padrão e moda de 17 anos. Para análise de risco, as jovens foram agrupadas em maiores e menores de 15 anos, considerando a idade de 15 anos como a inicial de relação sexual apresentada por Berquó ${ }^{18}$ para o Brasil.

A partir das respostas obtidas pelo questionário foram criadas categorias, usando a Técnica de Análise de Conteúdo de $\operatorname{Bardin}^{19}$; após isto, elas foram analisadas através dos recursos do Programa Epi Info versão 3.3.2. Foram usados os testes estatísticos odds ratio e risco relativo para verificar a associação entre variáveis, com intervalo de confiança de $95 \%$.

\section{Resultados}

A amostra estudada demonstrou 559 jovens com vida sexual ativa, encontrando-se a referência de 149 abortamentos provocados. Das 559 adolescentes, 296 (53\%) moravam com os pais, 474 $(84,8 \%)$ não trabalhavam, $182(32,6 \%)$ já haviam engravidado e 149 (26,7\%) informaram ter provocado aborto (Tabela 1 ). 
Encontrou-se um percentual de 9,7\% das jovens menores de 15 anos com vida sexual ativa, e dentre estas 7,4\% faziam parte do grupo que já havia provocado aborto (Tabela 1). Encontrouse um odds ratio de $0,21\left(\mathrm{IC}_{95 \%}=0,11\right.$ a 0,36$)$ e um risco relativo de $0,22\left(\mathrm{IC}_{95 \%}=0,13\right.$ a 0,38$)$, significativos e protetores para abortar antes dos 15 anos de idade (Tabela 2). O número de abortos foi maior nas escolas públicas $(69,8 \%)$ (Tabela 3 ), sendo significativa a relação entre abortar e estudar em escolas públicas com odds $=1,41$ $\left(\mathrm{IC}_{95 \%}=1,01\right.$ a 2,06$)$ e $\mathrm{RR}=1,44\left(\mathrm{IC}_{95 \%}=1,01\right.$ a 1,98$)$ (Tabela 2).

Diversos foram os motivos apontados pelas adolescentes para praticarem o aborto. Algumas delas citaram apenas um motivo $(32,2 \%)$, ou- tras mencionaram dois $(65,1 \%)$ ou três $(2,7 \%)$ associados (Tabela 3 ).

O motivo mais citado foi o medo da reação dos pais $(57,7 \%)$ quando soubessem da gravidez, esteja este motivo apontado como único $(22,1 \%)$ ou associado a outros, tais como: "sou muito nova, é minha idade, filho cedo limita" $(16,8 \%)$, associado à questão de o companheiro não ter aceitado a gravidez, nem o filho (16,1\%), ou associado aos outros dois motivos anteriores $(2,7 \%)$. O segundo motivo mais citado foi idade $(43,5 \%)$, seja como motivo único $(0,7)$, seja junto a outros: "não desejava essa gravidez" (14,1\%), "meu namorado não queria a gravidez, ele não quis o filho" (10,1\%), associado ao medo do que iriam falar $(4,7 \%)$ associado à falta de apoio da

Tabela 1. Distribuição das adolescentes estudadas segundo escolaridade, residência, trabalho, gravidez e abortamento - Maceió (AL), 2005.

\begin{tabular}{|c|c|c|c|c|c|c|}
\hline \multirow{3}{*}{ Características } & \multicolumn{4}{|c|}{ Idade (anos) } & \multirow{2}{*}{\multicolumn{2}{|c|}{$\begin{array}{c}\text { Total } \\
\mathrm{n}=559\end{array}$}} \\
\hline & \multicolumn{2}{|c|}{$12-14$} & \multicolumn{2}{|c|}{$15-19$} & & \\
\hline & $\mathrm{n}=54$ & $9,7 \%$ & $\mathrm{n}=505$ & $90,3 \%$ & $\mathbf{n}$ & $\%$ \\
\hline \multicolumn{7}{|l|}{ Escolaridade } \\
\hline Fundamental & 53 & 33,3 & 106 & 66,7 & 159 & 28,4 \\
\hline Médio & 1 & 0,2 & 399 & 99,8 & 400 & 71,6 \\
\hline \multicolumn{7}{|l|}{ Residência } \\
\hline Ambos os pais & 37 & 12,5 & 259 & 87,5 & 296 & 53,0 \\
\hline Somente com a mãe & 14 & 9,1 & 140 & 90,9 & 154 & 27,9 \\
\hline Somente com o pai & - & - & 7 & 100,0 & 7 & 1.3 \\
\hline Companheiro & 2 & 3,3 & 59 & 96,7 & 61 & 10,9 \\
\hline Outros & 1 & 2,4 & 40 & 97,6 & 41 & 7,3 \\
\hline \multicolumn{7}{|l|}{ Trabalho } \\
\hline Sim & 4 & 4,7 & 81 & 95,3 & 85 & 15,2 \\
\hline Não & 50 & 10,5 & 424 & 89,5 & 474 & 84,8 \\
\hline \multicolumn{7}{|l|}{ Gravidez } \\
\hline Sim & 13 & 7,2 & 168 & 92,8 & 181 & 32,4 \\
\hline Não & 41 & 10,8 & 337 & 89,2 & 378 & 67,6 \\
\hline \multicolumn{7}{|l|}{ Aborto } \\
\hline Sim & 11 & 7,4 & 138 & 92,6 & 149 & 26,7 \\
\hline Não & 43 & 10,5 & 367 & 89,5 & 410 & 73,3 \\
\hline
\end{tabular}

Tabela 2. Análise de riscos - Maceió (AL), 2005.

\begin{tabular}{llcccc}
\hline Variável dependente & Variável independente & $\mathbf{R}$ & $\mathbf{I C}_{95 \%}$ & OR & IC $_{95 \%}$ \\
\hline Idade 12-14 anos & Aborto & $0,22^{*}$ & 0,13 a 0,38 & $0,21^{*}$ & 0,11 a 0,36 \\
Idade 15-19 anos & União marital & $3,31^{* *}$ & 2,92 a 3,75 & $3,59^{* *}$ & 8,05 a 22,9 \\
Escola pública & Aborto & $1,41^{* *}$ & 1,01 a 1,98 & $1,44^{* *}$ & 1,01 a 2,08 \\
\hline
\end{tabular}

$\mathrm{RR}=$ risco relativo; $\mathrm{OR}=$ odds ratio.

*Significativo protetor; ${ }^{*}$ Significativo promotor. 


.

Tabela 3. Distribuição das adolescentes por motivos para abortar e tipo de escolas onde estudavam - Maceió (AL), 2005.

\begin{tabular}{|c|c|c|c|c|c|c|c|c|c|c|}
\hline \multirow{3}{*}{ Motivos } & \multicolumn{4}{|c|}{ Tipo de escola } & \multicolumn{4}{|c|}{ Idade } & & \\
\hline & \multicolumn{2}{|c|}{ Pública } & \multicolumn{2}{|c|}{ Particular } & \multicolumn{2}{|c|}{$12-14$} & \multicolumn{2}{|c|}{$15-19$} & \multicolumn{2}{|c|}{ Total } \\
\hline & $\mathrm{n}=104$ & $69,8 \%$ & $\mathrm{n}=45$ & $30,2 \%$ & $\mathrm{n}=13$ & $8,7 \%$ & $\mathrm{n}=136$ & $91,3 \%$ & 149 & $\%$ \\
\hline Medo da reação dos pais & 19 & 57,6 & 14 & 42,4 & 5 & 15,2 & 28 & 84,8 & 33 & 22,1 \\
\hline Medo da reação dos pais/Idade & 15 & 60,0 & 10 & 40,0 & 1 & 4,0 & 24 & 96,0 & 25 & 16,7 \\
\hline $\begin{array}{l}\text { Medo dos pais/Companheiro } \\
\text { não quis o filho }\end{array}$ & 17 & 70,8 & 7 & 29,2 & 1 & 4,7 & 23 & 95,8 & 24 & 16,1 \\
\hline Idade/Não queria engravidar & 13 & 61,9 & 8 & 38,1 & 4 & 19,4 & 17 & 80,9 & 21 & 14,1 \\
\hline $\begin{array}{l}\text { Idade/Companheiro não quis o } \\
\text { filho }\end{array}$ & 13 & 86,7 & 2 & 13,3 & 1 & 6,7 & 14 & 93,3 & 15 & 10,1 \\
\hline Companheiro não quis o filho & 12 & 92,3 & 1 & 7,7 & 1 & 7,7 & 12 & 92,3 & 13 & 8,7 \\
\hline Idade/Medo do que iam falar & 7 & 100 & - & - & - & - & 7 & 100 & 7 & 4,7 \\
\hline Idade/Mãe não apoiou a gravidez & 4 & 80,0 & 1 & 20,0 & - & - & 5 & 100 & 5 & 3,4 \\
\hline $\begin{array}{l}\text { Medo da reação dos pais/Idade/ } \\
\text { Companheiro não quis o filho }\end{array}$ & 2 & 50,0 & 2 & 50,0 & - & - & 4 & 100 & 4 & 2,7 \\
\hline Idade & 1 & 100 & - & - & - & - & 1 & 100 & 1 & 0,7 \\
\hline Estupro & 1 & 100 & - & - & - & - & 1 & 100 & 1 & 0,7 \\
\hline
\end{tabular}

mãe $(3,4 \%)$, ou as situações já citadas de associação com o medo dos pais $(16,8 \%)$, ou ainda com medo dos pais e rejeição do companheiro $(2,7 \%)$. A violência contra a mulher também aparece em pequena escala. O estupro aparece como motivo para abortar por uma única jovem estudante de escola pública $(0,7 \%)$, sendo esta uma das situações legalmente aceitas para a prática do aborto no Brasil (Tabela 3).

Ao se observarem os motivos distribuídos por faixa etária (Tabela 3 ), verifica-se que o motivo mais frequente continua sendo o medo da reação dos pais, quer seja na faixa das menores de 15 anos, quer nas mais velhas. Alguns motivos foram citados apenas pelas maiores de 15 anos, tais como a idade e a junção de três fatores: medo dos pais, idade e o companheiro não querer o filho.

Não se encontrou significância entre os motivos e a idade, e entre os motivos e o tipo de escola.

\section{Discussão}

A coleta de informações sobre aborto provocado no Brasil é prejudicada, tendo em vista a sua condenação por preceitos éticos, morais e religiosos. Isso dificulta os estudo sobre freqüência, estando o sub-registro presente tanto nos contextos em que o ato é legal (nas duas situações em que a prática é garantida por lei, ou seja, nos casos de estupro e de saúde da mãe) e principalmente nos outros casos que são considerados ilegais ${ }^{16}$.
Combinações de metodologias têm sido apontadas como caminhos para o estudo do aborto induzido em países com leis restritivas ${ }^{15,16}$. As diferentes medidas utilizadas pelos pesquisadores e as amostras diferenciadas dificultam a comparação dos resultados ${ }^{16}$.

Os fatores citados e o tipo de instrumento utilizado, ou seja, o questionário, possibilitam a visualização das limitações a que este estudo está submetido. Apesar de se trabalhar com uma amostra representativa, o local escolhido para a coleta de dados direciona para um ambiente em que as adolescentes apresentam características diferentes daquelas que estão fora da escola, ou em processo de exclusão $\mathrm{o}^{20-22}$.

O uso do questionário autoaplicável no estudo de temas difíceis de serem verbalizados, como é o caso do aborto provocado, traz à tona dois aspectos para reflexão: um é a possibilidade de facilitar a confissão do fato, pela garantia do anonimato. Caso que aconteceu em um dos questionários no qual a jovem expôs a sua gratidão, em poder "desabafar" o ato realizado, que a estava "amargurando há quinze dias" (sic). O outro aspecto é a possibilidade de não fornecer o número real de acontecimentos, em razão da omissão de algumas adolescentes pesquisadas em falar sobre o ato realizado.

A facilidade ou não em falar sobre sexualidade relaciona-se com questões culturais, tais como a sociedade estudada encara o sexo e sua prática. Apesar de se observar a exaltação da sensualidade na mídia brasileira, essa sociedade continua 
com alguns valores antigos, principalmente quanto ao início da vida sexual genital para as mulheres $^{18,20,23}$. Isto pode ser um viés nos dados encontrados neste estudo, no qual ocorreu maior frequência de casos de aborto provocado nas escolas públicas $(69,8 \%)$ (Tabela 3), apesar dos riscos significativos encontrados $(\mathrm{RR}=1,41$; $\mathrm{IC}_{95 \%}=1,01$ a $1,98 /$ Odds $=1,44 ; \mathrm{IC}_{95 \%}=1,01$ a 2,08$)$ (Tabela 2).

Orientações educativas, como em qualquer outro aspecto, servem como fator de proteção para os riscos de uma vida sexual sem prevenção $0^{3,8-11}$. Apesar de existirem ações nesse âmbito no Brasil $^{14,20-24}$, as estatísticas brasileiras mostram que está ocorrendo o aumento de gravidez na faixa etária dos 10 aos 14 anos de idade ${ }^{12,23,24}$. Tal dado aponta para a necessidade de se iniciar mais cedo essa orientação nas escolas, visando prevenir os riscos de uma vida sexual e reprodutiva sem as informações e orientações adequadas ${ }^{3-7,22-27}$.

A gravidez na adolescência vem sendo apontada como uma das razões do abandono da escola pelas jovens ${ }^{21,23,26,27}$. Fatores como escolaridade e renda têm relação com essa decisão, sendo proporcionalmente inversos à idade ${ }^{21-24}$. A maneira como ocorre a primeira informação às jovens sobre reprodução e contracepção permite identificar as formas de socialização para a sexualidade por elas vivenciada ${ }^{3,14,22,26}$.

Todas as adolescentes deste estudo estão na escola, o que aponta para uma situação diferente daquela que geralmente associa a gravidez ao abandono dos estudos, e ao desconhecimento dos aspectos sobre a sexualidade e saúde reprodutiva $^{22-27}$. As componentes dessa amostra, diferente da literatura sobre o assunto ${ }^{4,14,23,26}$, vivem com ambos os pais, não trabalham e são solteiras (Tabela 3), o que recorda os $86 \%$ de adolescentes solteiras grávidas na cidade de Maceió em $2004^{13}$, salientando a dificuldade em revelar a prática do aborto ante a sua ilegalidade ${ }^{16}$.

Novamente, destaca-se a questão do tipo de instrumento usado para a coleta dos dados. Acredita-se que a garantia de seu anonimato possibilitou a obtenção das revelações, dos desabafos, que geralmente são difíceis de conseguir por outra forma, quando não se tem uma proximidade e confiança, considerando-se tabus e questões culturais associados ao aborto provocado ${ }^{4,11,25}$.

Segundo Brandão ${ }^{20}$, o modo como adolescentes brasileiras enfrentam as primeiras suspeitas de estarem grávidas está associado a duas situações: uma dependente do relacionamento prévio com os pais (da possibilidade de diálogo entre gerações e da atitude dos pais em relação à sexualidade) e outra do relacionamento existente entre os parceiros envolvidos.

A inadequação dos serviços de saúde para atender às adolescentes e o desconhecimento delas sobre os aspectos relacionados à sua saúde reprodutiva são aspectos relevantes nesse contexto, quando se pensa na prevenção $0^{4,14,21}$. Agregados a esses aspectos, o exercício irregular das relações sexuais e a alternância dos ciclos menstruais, geralmente irregulares na faixa etária dos 12-15 anos, fazem com que algumas jovens tenham dificuldade em admitir que estejam grávidas antes do primeiro trimestre de gravidez. Este avanço na idade gestacional leva a uma situação maior de risco, caso a adolescente opte pelo aborto $^{12,17}$. Dessa forma, assumir ou não uma gravidez e o exercício de uma vida sexual ativa, com o uso de métodos contraceptivos, são situações de crescimento e aprendizado de responsabilidades e autonomia para as adolescentes.

A busca pelo aborto provocado $(26,7 \%$, Tabela 1) e os motivos mais frequentes encontrados neste estudo, ou seja, o medo da reação dos pais, idade e reação dos companheiros $(57,7 \%$, Tabela 3), apontam para a possível ausência de alguns dos aspectos reveladores de maturidade emocional.

Pelo fato de estarem frequentando uma escola, é possível que tenham recebido informações sobre os aspectos preventivos para saúde reprodutiva $^{22,23}$, questão que exige uma reflexão sobre a eficácia dessas informações na prevenção da gravidez não planejada e o consequente abortamento que aparecem neste estudo (26,7\%, Tabela 1$)$.

A família e a escola são importantes na transmissão de informações aos jovens ${ }^{18,20}$. No Brasil, as desigualdades sociais se refletem na qualidade e no acesso ao sistema educacional, fato que se verifica na significância estatística encontrada neste estudo, bem como na citação maior de abortos nas escolas públicas estudadas (Tabelas $2 \mathrm{e}$ 3). Reconhece-se que este último dado, ou seja, a menor frequência de citações do aborto nas escolas particulares, deva-se em parte à dificuldade em falar sobre a sexualidade e seus aspectos, o que pode ter ocorrido nessas escolas. Sabe-se que quanto maiores forem as censuras internas e os tabus sobre o tema, como é o caso do tema aqui estudado, maiores serão os entraves para sua verbalização $o^{4,6}$.

A partir dos dados aqui encontrados, e considerando-se a transmissão de informações sobre educação sexual ${ }^{3,20}$, que acontece de forma transversal nas escolas brasileiras, destaca-se a necessidade de maior atenção a essas ações, bem como 
àquelas que se preconizam no âmbito das políticas públicas na área da saúde do adolescente ${ }^{23}$.

Assumir responsabilidades é um ato que ainda está sendo aprendido nessa fase; portanto, assumir a tomada de decisão é difícil2,10,20. Essa situação é visualizada nos motivos aqui encontrados para o abortamento, que ao serem analisados, mostram apenas $21(14,1 \%)$ das adolescentes admitindo haver abortado por não desejarem a gravidez. Todos os outros motivos foram relacionados ou associados à reação que outras pessoas poderiam ter, sendo os pais, companheiros e relações sociais elementos importantes nesse contexto (Tabela 3).

A dependência dos jovens aos pais é elemento importante neste estudo, seja ela sentimental ou econômica. Observa-se que o medo da reação dos genitores é um sentimento presente na maioria dos motivos relatados, seja ele o único ou associado a outro motivo como a idade (Tabela 3).

Estudos brasileiros sobre gravidez na adolescência apontam para o fato de que as jovens que contam com o apoio dos pais e a possibilidade de diálogo com eles sobre sexo começam a vida sexual mais tarde $e^{4,6}$. Caso engravidem, conseguem mediante o diálogo tomarem uma atitude que melhor se enquadre à sua situação, diminuindo os traumas de um abortamento provocado, a manutenção da gravidez e o não abandono da escola ${ }^{4,14,21}$.

Os dados encontrados neste estudo referentes a abortamentos antes dos 15 anos de idade corroboram os estudos brasileiros ${ }^{25,26}$. Pesquisa realizada pelo Instituto $\operatorname{Ecos}^{26} \mathrm{em}$ cinco capitais brasileiras apresentou dados do Datasus, entre os anos de 1995 e 2000, que revelaram uma tendência de crescimento no percentual de abortos admitidos na rede de saúde ${ }^{25}$.

O fato de ser significativa a relação entre estudar em escolas públicas e praticar o aborto chama a atenção para questões sociais, ao se considerar que o acesso ao sistema escolar brasileiro, assim como aos serviços de saúde, é determinado pela distribuição de renda ${ }^{14,25-27}$.

No Brasil, existem grandes diferenças sociais e econômicas nas trajetórias dos jovens. Se, por um lado, uma parcela da população jovem fica excluída de seus direitos de cidadãos, tais como moradia, escola, saúde e lazer, por outro, grupos sociais mais favorecidos apresentam um prolongamento da juventude com o aumento do tempo de estudo e a manutenção da coabitação com os pais e atraso da autonomia financeira ${ }^{21,22}$.

\section{Conclusão}

Tendo em vista os dados encontrados como causa de abortamento nessa população, o medo da reação dos pais, a idade, a falta de apoio do companheiro ou da mãe, a rejeição em si da gravidez, bem como a relação significativa de abortamento em escolas públicas em relação às particulares, verifica-se que, apesar da proibição legal e das políticas públicas de educação e de saúde, o aborto provocado continua acontecendo no Brasil, mesmo quando considerada a população menor de 15 anos.

Os motivos aqui encontrados para a prática do aborto destacam a importância da comunicação sobre os diversos aspectos da sexualidade, principalmente no âmbito familiar e educacional formal.

Visualiza-se a necessidade de maior esclarecimento aos jovens e aos seus pais sobre a saúde reprodutiva dos jovens. O medo da reação dos pais pode demonstrar um afastamento na relação entre as gerações, o que sugere a realização de novos estudos nesse âmbito.

\section{Colaboradores}

DS Correia trabalhou na concepção, no delineamento, análise e interpretação dos dados e na redação do artigo; JC Cavalcante, na análise estatística dos dados; EST Egito, na revisão crítica; EMC Maia trabalhou no delineamento da pesquisa, na revisão crítica e na aprovação da versão a ser publicada. 


\section{Agradecimentos}

À Fundação de Apoio a Pesquisa do Estado de Alagoas (Fapeal), pelo apoio financeiro ao projeto; à professora Maria Jésia Vieira, pelo apoio nas discussões do trabalho.

\section{Referências}

1. Brasil. Ministério da Saúde. Secretaria de Atenção à Saúde. Área de Saúde do Adolescente e do Jovem. Marco legal: saúde, um direito de adolescentes. Brasília: Ministério da Saúde; 2005.

2. Correia DS. Adolescentes no trânsito: perigo à vista? Maceió: Catavento; 2000.

3. Altmann H. Educação sexual e primeira relação sexual: entre expectativas e prescrições. Rev Estud Fem [periódico na internet]. 2007 maio-ago [acessado 2008 jan 10]; 15(2):[cerca de 23 p.]. Disponível em: http://www.scielo.br/pdf/ref/v15n2/a04v15n2pdf

4. Peres SO, Heilborn ML. Cogitação e prática do aborto entre jovens em contexto de interdição legal: o avesso da gravidez na adolescência. Cad Saude Publica 2006; 22(7):1411-1420.

5. Allan Guttmacher Institute. Abortion [documento na Internet] [cited 2007 Jan 22]. Available from: http://www.guttmacher.org/sections/abortion.php

6. Cabral CS. Gravidez na adolescência: negociações na família. In: Heilborn ML, Duarte LFD, Peixoto C, Barros ML, organizadores. Sexualidade, família e ethos religioso. Rio de Janeiro: Garamond; 2005. p. 87-110.

7. World Health Organization. Sexual relations among young people in developing countries 2001. [cited 2006 May 1] [about 66 pages ]. Available from: http:// www.who.int/reproductive-health/publications RHR_01_8/index.html

8. Sedgh G, Henshaw S, Singh S, Åhman E, Shah IH. Induced abortion: estimated rates and trends worldwide. Lancet 2007; 370(9595):338-1345.

9. Sihvo S, Bajos N, Ducot B, Kaminski M. Women's life cycle and abortion decision in unintended pregnancies. JECH 2003; 57(8):601-605.

10. Widman L, Welsh DP, McNulty JK, Little KC. Sexual communication and contraceptive use in adolescent dating couples. J Adolesc Health 2006; 39:893-899.

11. Harvey N, Gaudoin M. Teenagers requesting pregnancy termination are no less responsible about contraceptive use at the time of conception than older women. BJOG 2007; 114:226-229.

12. Sousa MH, Cecatti JG, Hardy EE, Amaral E, Souza JPD, Serruya S. Sistemas de informação em saúde e monitoramento de morbidade materna grave e mortalidade materna. Rev Bras Saúde Mater Infant 2006; 6(2):161-168.

13. Brasil. Ministério da Saúde. Datasus. Informações de Saúde. [documento na Internet] [acessado 2006 mar 6]. Disponível em: http://w3.datasus.gov.br/ datasus/datasus.php?area

14. Menezes GMS, Aquino EML, Silva DO. Aborto provocado na juventude: desigualdades sociais no desfecho da primeira gravidez. Cad Saude Publica 2006; 22(7):1431-1446.
15. Rossier C. Estimating induced abortion rates: a review. Studies in Family Planning 2003; 34(2):87-102.

16. Olinto MTA, Moreira Filho DC. Estimativa de aborto induzido: comparação entre duas metodologias. Rev Panam Salud Publica/Pan Am J Public Health 2004; 15(5):331-336.

17. Corrêa S, Freitas A. Voluntary interruption of the pregnancy in Brazil. Rev Estudos Feministas 1997; 2:389-395.

18. Berquó E, coordenadora. Comportamento sexual da população brasileira e percepções do HIV/Aids. Brasília: Ministério da Saúde; 2000.

19. Bardin L. Análise de conteúdo. Lisboa: Edições 70; 1977.

20. Brandão ER. Revelação da gravidez na adolescência em famílias de camadas médias: tensões e dilemas. In: Heilborn ML, Duarte LFD, Peixoto C, Barros ML, organizadores. Sexualidade, família e ethos religioso. Rio de Janeiro: Garamond; 2005. p. 111-134.

21. Villela WV, Doreto DT. Sobre a experiência sexual dos jovens. Cad Saude Publica 2006; 22(11):2467-2472.

22. Altmann H. A sexualidade adolescente como foco de investimento político-social. Educ Rev 2007; 46:287-310.

23. Brasil. Ministério da Saúde. Secretaria de Atenção à Saúde. Departamento de Ações Programáticas Estratégicas. Marco teórico e referencial: saúde sexual e saúde reprodutiva de adolescentes e jovens. Brasília: Ministério da Saúde; 2006.

24. Brasil. Ministério da Saúde. Planejamento familiar: manual para o gestor. Brasília: Ministério da Saúde; 2002.

25. Vieira LM, Goldberg TBL, Saes SO, Dória AAB. Abortamento na adolescência: um estudo epidemiológico. Cien Saude Colet 2007; 12(5):1201-1208.

26. Ecos Comunicação em Sexualidade. Gravidez de adolescentes entre 10 e 14 anos e vulnerabilidade social: estudo exploratório em cinco capitais brasileiras. Rio de Janeiro: Ecos; 2004.

27. Ferraro AR, Machado NCF. Da universalização do acesso à escola no Brasil. Educ Soc 2002; 23(79):213214 .

Artigo apresentado em 06/08/2008

Aprovado em 26/11/2008

Versão final apresentada em 08/12/2008 